\title{
Another chemokine target bites the dust?
}

\section{Roberto Solari}

Scanning through the table of contents in Thorax, you might be forgiven for skipping over the paper by Wang et $a l^{1}$ Redundancy in the chemokine field may not feel like news; however, I would suggest you pause and take time to read the article and perhaps reflect on a number of important issues that this study highlights. My reflections are about publishing negative data, the role of CCR8 in asthma and some general principles about drug discovery.

\section{THE IMPORTANCE OF NEGATIVE STUDIES}

The paper by Wang is somewhat unusual because it essentially reports a negative conclusion. Judging by the number of internet blogs on the topic, it seems there is genuine concern about the inability to publish negative results, and there is now quantitative evidence to suggest that there is a growing trend for journals to publish studies with positive outcomes. ${ }^{2}{ }^{3}$ There may be many reasons for this bias, including increased competition for research funding and pressure on scientists who are evaluated by the number of publications and their citations combined with the fact that positive studies receive more citations than negative studies. Whether this is a healthy trend or is undermining science is an important debate, and there is a movement by open access journals to redress the bias. Interestingly, Wang and colleagues have, either deliberately or subconsciously, written the title of their paper in the affirmative to give their study the appearance of a positive outcome.

Why are negative studies of value to the community? Many of us were trained as scientists according to the principles of falsifiability as described by Popper. His famous 'black swan' example is one we are probably all very familiar with. Science philosophy has moved on from Popper and most laboratory scientists realise that interpreting negative data is never quite as black and white as swans; nevertheless, a well-designed and wellexecuted experiment that falsifies a

Correspondence to Dr Roberto Solari, GlaxoSmithKline, Respiratory Therapy Area, National Heart and Lung Institute, Imperial College, Norfolk Place, London W2 1PG, UK; r.solari@imperial.ac.uk hypothesis or supports a null hypothesis is integral to science. ${ }^{4}$ It can also reduce the waste of precious time and resources and unnecessary animal experimentation and human studies. Some in academia argue that reporting negative results has much less impact than 'paradigm shifting' positive results; however, in the pharmaceutical industry learning from negative results has become a mantra. At every step along the drug discovery pipeline, potential drugs will fail for a variety of reasons and much focus in recent years in the industry has been to increase efficiency by reducing this expensive and time-consuming attrition. The later a drug fails, the more money and time has been invested; consequently, it is accepted wisdom for project managers to try to design critical go/no-go decision points as early in the process as they can. In drug discovery pipelines, learning from failure has become as important as 'paradigm shifting' breakthroughs both to facilitate progress and to kill doomed projects quickly and cheaply.

\section{CCR8 AS AN ASTHMA TARGET}

Producing new drugs for asthma is a difficult business. The current medicines have been with us for a very long time, and physicians now have decades of experience in using what are relatively cheap, safe and, for most patients, efficacious products. That is not to say that current therapeutic options are perfect or that there is no longer any unmet need, but there are substantial hurdles to making a novel medicine that addresses those unmet needs. Much of asthma research over the past 25 years has been dominated by the Th2 hypothesis, which proposed an excessive Th2 response to normally innocuous inhaled antigens to be at the heart of the immune and inflammatory pathology. This dominant view of asthma grew to a large extent from mouse immunology and has led many researchers to explore the therapeutic potential of blocking the mediators that drive this overexuberant Th2 response. So what made CCR8 an asthma target in the first place and can we learn anything from this process that can enlighten us when choosing the next novel targets? CCR 8 is a receptor for the chemokine CCL1 and is expressed on Th2 cells, alongside two other chemokine receptors CCR3 and CCR4. Chemokine receptors are members of the $G$ protein-coupled receptor family (GPCR), which represent the most successful class of drug targets for the pharmaceutical industry. So that ticks two boxes: the target is on the 'right' cell type (if you are an advocate of the Th2 hypothesis) and it is probably 'druggable'. Mouse models gave conflicting results with some studies showing CCR8/CCL1 was important for driving an inflammatory response to an inhaled allergen, ${ }^{5}$ whereas other studies showed no impact. $^{6-8}$ Human studies showed increased levels of CCR1 and CCR $8+T$ cells in the lungs of asthmatics, showing a correlation with the disease. ${ }^{9} 10$ The mouse data are clearly contradictory and the human data do not prove causality, but because of our intrinsic bias towards positive data, we might conclude that, on balance, it looks like a CCR8 antagonist might be a useful medicine for asthma. But knowing that it is going to cost tens of millions of dollars to take a molecule from discovery to a proof-of-concept study in humans, do you have enough information to initiate the programme? Before you press the start button, there are perhaps a few more questions that you would like to ask. The first is whether CCR8/CCL1 is both sufficient and necessary to drive Th2 chemotaxis. Remember Th2 cells have more than one chemokine receptor and the Mikhak study suggests CCR4 is the dominant Th2 chemotaxis receptor, in mice at least. ${ }^{8}$ The second is what level of confidence do we have that blocking Th2 chemotaxis will deliver therapeutic benefit in human asthma and if so why will a CCR8 antagonist be better than or differentiated from current medicines? Just making a different new drug is not enough these days, neither for patients nor for payers. Finally, what is the quickest, cheapest and most reliable point at which you can make these decisions, remembering that it is usually much easier to start than to stop programmes?

So what has the Wang paper taught us about all this? The group has produced a CCR8 antagonist with acceptable pharmacological properties for in vivo dosing and hypothesis testing-a major achievement in itself that will have taken many years of hard effort and substantial cost. However, administering the drug at a dose that was sufficient to achieve receptor blockade had no effect on lung symptoms following inhaled allergen challenge. If one believes that the Ascaris-sensitised cynomolgus monkey model is predictive of efficacy in human asthma, then there is only one 
logical conclusion-CCR8 is not a good asthma target. However, if you had a room full of asthma experts, I doubt there would be consensus as to the value of this model as a predictor of human efficacy. So, should one place more weight on a negative primate model than a positive rodent model, or are we placing too much weight on animal models both for selecting targets and in this case, for validating a target? It seems to me that using an unreliable or unproven decision-making experiment after spending many years of research and millions of dollars is not a productive way forward for the drug discovery industry. CCR8 may still be a good drug target in certain patients and in certain indications. Although the Wang paper is an excellent and careful study and tells us something about Th2 recruitment following allergen challenge, I am not sure it really helps us out with the bigger questions about human disease. We need to be careful about asking the right questions and focusing on the unmet medical need and how a new medicine might be used to address it before we start or stop a drug discovery project. It also raises an issue of animal ethics. If one does not believe a primate or rodent model is predictive of human efficacy, then why do it? Animal models are mandatory for safety and toxicology studies, but efficacy models are not. They are undoubtedly useful in studying mechanisms and for determining potentially active and safe doses in human studies, but there is still a box ticking mentality to animal disease models when most people would question their predictive power. ${ }^{11}$

\section{CHEMOKINES AND DRUG DISCOVERY}

Chemokines were discovered in the 1980s. Because of their involvement in inflammation and the fact that their receptors are GPCRs, they have long been considered attractive targets. There has been a substantial investment in drug discovery efforts in looking for antagonists that to date has yielded two approved medicines: Plerixafor-a CXCR4 antagonist used for stem cell mobilisation; and Maraviroc-a CCR5 antagonist for blocking HIV entry into cells. There are many chemokine drugs in clinical trials, both small molecules and antibodies, and many agents have already fallen by the wayside, often late in development when huge amounts of money and effort have been spent. ${ }^{12}$ There is an opinion that chemokines have not delivered on their drug discovery promise and that chemokine receptors are particularly difficult drug targets. Three main reasons have been proposed to explain this apparent lack of productivity. ${ }^{13} 14$ First, the immune and inflammatory systems of model animal species and humans have significant differences, which makes selecting and validating targets particularly challenging. Second, the chemokine system has often been described as highly redundant, that is, the receptors can be promiscuous, binding several ligands, and ligands can bind several receptors. Moreover, immune cells often express multiple chemokine receptors. The common interpretation of this is that multiple chemokines can perform the same physiological function and therefore antagonism of one receptor will never be sufficient. Third, drug trials often fail to achieve sufficiently high plasma levels of the drug to achieve sufficient receptor blockade to demonstrate efficacy. ${ }^{14}$

Both Horuk ${ }^{13}$ and Schall ${ }^{14}$ hypothesise that in order to see efficacy, more than $90 \%$ of chemokine receptors need to be occupied by the antagonist and in most studies this level is not reached. The Wang paper used a pharmacodynamic assay to measure CCR8 occupancy on CD4 T cells in whole blood following dosing with drug by measuring competition for binding of a labelled CCL1 ligand. They clearly show that at all time points in the study the CCR8 antagonist drug exceeded 98\% receptor occupancy-so the first challenge was met.

Horuk makes a case for chemokine and receptor redundancy as a complicating factor and has proposed a solution of promiscuous or dual-specific antagonists to inhibit multiple receptors. ${ }^{13}$ Schall and Proudfoot make it clear that they believe chemokine redundancy is a fallacy and that the apparent overlapping functions of chemokines reflect our limited ability to perform relevant functional assays. ${ }^{14}$ They propose that each chemokine-receptor system has a specific role in a particular physiological and pathological context. It is an interesting hypothesis, but one that is difficult to assess pre-clinically. If it means one has to wait until proofof-concept trials in humans for an answer, this makes chemokine drug discovery a risky venture indeed. Much has been made of species differences in the chemokine system; however, in my opinion this is no different to any other drug discovery programme, where care is always taken to explore the species-specific pharmacology and physiology, and reinforces the importance of using human samples wherever possible.

My view is that the chemistry of chemokine receptor antagonists is challenging. Discovering and developing small molecules with good drug-like properties have been a significant problem and many failures may be more compound related rather than mechanism related. Second, I believe we have developed an overreliance on animal models to identify targets and to test our compounds. If, as we assume from the Wang paper, this CCR8 antagonist is terminated, then it is because of the lack of efficacy in this Ascaris challenge model, which must mean there is a high degree of confidence that this is predictive of efficacy in human asthma. Asthma, like most chronic inflammatory diseases, is complex in its origins and pathology, and most researchers now accept the need to stratify patients and specifically target the remaining unmet need. The animal models that we have developed have undoubtedly provided much of our mechanistic insights into the basic processes involved in asthma and they certainly worked well for corticosteroids. However, they are very poorly predictive of efficacy for other classes of drugs. ${ }^{11}$ Once again, our bias for positive data outweighs all the negative data. This is an active debate that is crucial if we are to improve our success rate in developing new medicines for asthma. Reading the Wang paper may motivate you to take part in the debate.

\section{Competing interests None.}

Provenance and peer review Commissioned; internally peer reviewed.

To cite Solari R. Thorax 2013;68:501-503.

Received 14 January 2013

Accepted 15 January 2013

Published Online First 5 March 2013

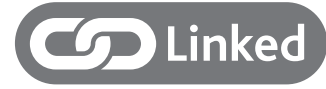

- http://dx.doi.org/10.1136/thoraxjnl-2012-203012

Thorax 2013;68:501-503.

doi:10.1136/thoraxjnl-2012-203167

\section{REFERENCES}

1 Wang $L$, Jenkins T, Dai M, et al. Antagonism of chemokine receptor CCR8 is ineffective in a primate model of asthma. Thorax 2013;68: 506-12.

2 Fanelli D. Do pressures to publish increase scientists' bias? An empirical support from US states data. PLoS ONE 2010;5:e10271.

3 Fanelli D. Negative results are disappearing from most disciplines and countries. Scientometrics 2012;90:891-904.

4 Browman HI. Negative results. Mar Ecol Prog Ser 1999;191:301-9.

5 Gonzalo J-A, Qiu Y, Lora JM, et al. Coordinated involvement of Mast cells and T cells in allergic mucosal inflammation: critical role of the CC 
chemokine ligand 1:CCR8 axis. J Immunol 2007;179:1740-50.

6 Chung CD, Kuo F, Kumer J, et al. CCR8 is not essential for the development of inflammation in a mouse model of allergic airway disease. J Immunol 2003;170:581-7.

7 Goya I, Villares R, Zaballos A, et al. Absence of CCR8 does not impair the response to ovalbumin-induced allergic airway disease. J Immunol 2003;170:2138-46.

8 Mikhak Z, Fukui M, Farsidjani A, et al. Contribution of CCR4 and CCR8 to antigen-specific
Th2 cell trafficking in allergic pulmonary inflammation. J Allergy Clin Immunol 2009;123:

67-73.

9 Montes-Vizuet $R$, Vega-Miranda A, Valencia-Maqueda $E$, et al. CC chemokine ligand 1 is released into the airways of atopic asthmatics. Eur Respir J 2006;28:59-67.

10 Mutalithas K, Guillen C, Raport C, et al. Expression of CCR8 is increased in asthma. Clin Exp Allergy 2010:40:1175-85.

11 Holmes AM, Solari R, Holgate ST. Animal models of asthma: value, limitations and opportunities for alternative approaches. Drug Discov Today 2011;16:659-70.

12 Proudfoot AEI, Power CA, Schwarz MK Anti-chemokine small molecule drugs: a promising future? Expert Opin Investig Drugs 2010;19:345-55.

13 Horuk R. Chemokine receptor antagonists: overcoming developmental hurdles. Nat Rev Drug Discov 2009;8:23-33.

14 Schall TJ, Proudfoot AEl. Overcoming hurdles in developing successful drugs targeting chemokine receptors. Nat Rev Immunol 2011; 11:355-63. 